\title{
Direct Image Guided Targeting Versus MER in DBS Surgery
}

\author{
Kaptan $\mathbf{H}^{*}$ \\ Department of Neurosurgery, Dokuz Eylül University Faculty of Medicine, Izmir, Turkey
}

*Corresponding Author: Kaptan H, Department of Neurosurgery, Dokuz Eylül University Faculty of Medicine, Izmir, Turkey, Email: hulagukaptan@yahoo.com

\section{SHORT COMMUNICATION}

DBS (Deep Brain Stimulation) is a surgical procedure that provides significant benefits for various neurological conditions. Common targets are STN(subthalamic nucleus) and GPi (globus pallidus pars interna), the VIM (ventral intermedius nucleus), PPN (pedunculopontine nucleus).

Advanced PD (Parkinson's disease) stimulation of the STN reduces tremor, rigidity, and bradykinesia. Essence of the promise, the most important step toward involving with DBS outcomes remains careful and right patient selection. Then, $>30 \%$ of DBS failures can be attributed to the inappropriate indication for surgery.

DBS is in principle "reversible lesion" in the target nucleus and does not interfere with the use of other therapies. STN is the main target nucleus for DBS in PD. STN stimulation directly improves and reduces off time, thereby reducing dopaminergic medication requirements by approximately $50 \%$; dyskinesias improve as a consequence.

MER (Micro Electrode Recordings), macrostimulation and anatomic lead placement with MRI or other image guidance is benefited in order to place the electrode correctly.

Intraoperative stimulation is more widely accepted as a tool for use during surgery, the debate is between MER and other methods. Due to hemorrhagic complications the use of MER during DBS operation was still questioning for some of surgeons.

Potential risk of MER; major vascular injury, hemorrhage; likely due to use of multiple parallel trajectory to reach the target nucleus. Other factors are awake surgery and the long duration of the operation.
Application of the FFT (Fast Fourier Transform) to MER data may be used for the decrease in the number of micro electrodes used for the surgery.

Therefore, a considerable discussion is currently ongoing with regard to the necessity for MER in DBS surgery.

The potential benefits of image guidance are: single-planned surgical targeting, shortening of surgical time and under general anesthesia. This approcah is very short time than MER. In addition; it should not be overlooked that intraoperative macro stimulation is important for clinical success. As a result of image guidance procedure; Less electrode transit is therefore less traumatic and postoperative side effect.

Variability in the position of the STN is important. For DBS; direct targeting, compared with indirect techniques; it may be more important to achieve the target.

\section{REFERENCES}

[1] Zrinzo L, Foltynie T, Limousin P. Reducing hemorrhagic complications in functional neurosurgery: a large case series and systematic literature review. J Neurosurg. 2012 Jan; 116(1):84-94. Epub 2011 Sep 9.

[2] Ashkan K, Blomstedt P, Zrinzo L, Tisch S, Yousry T, Limousin-Dowsey $\quad$ P, Hariz MI.Variability of the subthalamic nucleus: the case for direct MRI guided targeting. Br J Neurosurg. 2007 Apr;21(2):197-200.

[3] Kaptan H, Ekmekci H, Ayaz M. Deep Brain Stimulation In Parkinson Disease. Niger J Health Sci 2015;15:109-15.

[4] Ashkan K, Wallace B, Bell B et al . Deep brain stimulation of the subthalamic nucleus in Parkinson's disease 1993-2003: where are we 10 years on? Br J Neurosurg 2004;18:19-34

[5] Kaptan H,Çakmur R.Technical Case Report of Deep Brain Stimulation: Is it Possible Single Electrode Reach to Both of Subthalamic Nucleus and Ventral Intermediate Nucleus in 
One Stage? Open Access Maced J Med Sci. 2018 Apr 2;6(4):659-662.

[6] Ekmekçi H, Kaptan H. Camptocormia and deep brain stimulation: The interesting overlapping etiologies and the therapeutic role of subthalamic nucleus-deep brain stimulation in Parkinson disease with camptocormia. Surg
Neurol Int. 2016 Feb 10;7(Suppl 4):S103-7. doi: $10.4103 / 2152-7806.176130$

[7] Kaptan H, Ayaz M, Ekmekçi H. Effect Transformation of the Micro Electrode Recording (MER) Data to Fast Fourier Transform (FFT) for the Main Target Nucleus Determination for STN-DBS. Acta Inform Med. 2014 Dec 22(6): 411-412.

Citation: Kaptan H. Direct Image Guided Targeting Versus MER in DBS Surgery. ARC Journal of Neuroscience. 2018; 3(2):13-14. doi:dx.doi.org/10.20431/2456-057X.0302004.

Copyright: (C) 2018 Authors. This is an open-access article distributed under the terms of the Creative Commons Attribution License, which permits unrestricted use, distribution, and reproduction in any medium, provided the original author and source are credited. 